\title{
Failure to Consolidate the Consolidation Theory of Learning for Sensorimotor Adaptation Tasks
}

\author{
Graham Caithness, ${ }^{1}$ Rieko Osu, ${ }^{2}$ Paul Bays, ${ }^{3}$ Henry Chase,${ }^{3}$ Jessica Klassen, ${ }^{1}$ Mitsuo Kawato, ${ }^{2}$ Daniel M. Wolpert, ${ }^{3}$ and \\ J. Randall Flanagan ${ }^{1}$ \\ ${ }^{1}$ Department of Psychology and Centre for Neuroscience Studies, Queen's University, Kingston, Ontario, K7L 3N6 Canada, ${ }^{2}$ Advanced Telecommunications \\ Research Institute, Computational Neuroscience Laboratories, Kyoto 619-0288, Japan, and ${ }^{3}$ Sobell Department of Motor Neuroscience, Institute of \\ Neurology, University College London, London WC1N 3BG, United Kingdom
}

An influential idea in human motor learning is that there is a consolidation period during which motor memories are transformed from a fragile to a permanent state, no longer susceptible to interference from new learning. The evidence supporting this idea comes from studies showing that the motor memory of a task (A) is lost when an opposing task (B) is experienced soon after, but not if sufficient time is allowed to pass $(\sim 6 \mathrm{hr})$. We report results from three laboratories challenging this consolidation idea. We used an ABA paradigm in the context of a reaching task to assess the influence of experiencing B after A on the retention of A. In two experiments using visuomotor rotations, we found that B fully interferes with the retention of A even when B is experienced $24 \mathrm{hr}$ after A. Contrary to previous reports, in four experiments on learning force fields, we also observed full interference between A and B when they are separated by $24 \mathrm{hr}$ or even 1 week. This latter result holds for both position-dependent and velocity-dependent force fields. For both the visuomotor and force-field tasks, complete interference is still observed when the possible affects of anterograde interference are controlled through the use of washout trials. Our results fail to support the idea that motor memories become consolidated into a protected state. Rather, they are consistent with recent ideas of memory formation, which propose that memories can shift between active and inactive states.

Key words: motor learning; internal models; arm movement; visuomotor rotation; force field; retrograde interference

\section{Introduction}

Traditional theories of learning posit that new memories are transferred from a short-term labile state to a long-term fixed state that is more or less permanent and protected from new learning. The time course of this consolidation process after learning can be probed by having subjects learn new material and testing for retention of the original material. The process by which new learning may interfere with the consolidation of previous learning is referred to as retrograde interference. In human motor learning, support for such a consolidation process has been provided by two influential studies of dynamic motor learning (Brashers-Krug et al., 1996; Shadmehr and Brashers-Krug, 1997).

In these studies, subjects adapted to opposing force fields (A then $\mathrm{B}$ ) applied to the hand during a reaching task. When these fields were experienced $5 \mathrm{~min}$ apart, subjects showed no retention of A when tested the following day. However, when the fields were experienced $\geq 6 \mathrm{hr}$ apart, retention of A was observed. If only one field was experienced, retention was long lasting, per-

\footnotetext{
Received June 7, 2004; revised Aug. 4, 2004; accepted Aug. 19, 2004.

This work was supported by the Natural Sciences and Engineering Research Council of Canada, the Wellcome Trust, the National Institute of Information and Communications Technology (Japan), and the Human Frontier Science Program.

Correspondence should be addressed to Dr. J. R. Flanagan, Department of Psychology, Queen's University, Kingston, 0ntario, K7L 3N6 Canada. E-mail: flanagan@psyc.queensu.ca.

DOI:10.1523/JNEUROSCI.2214-04.2004

Copyright $\odot 2004$ Society for Neuroscience $\quad$ 0270-6474/04/248662-10\$15.00/0
}

sisting for as long as 5 months (Shadmehr and Brashers-Krug, 1997). These findings supported the idea that new motor memories undergo a period of consolidation, after which they become resistant to being overwritten by new learning. These studies have motivated many studies of motor learning and interference (Shadmehr and Holcomb, 1997; Krakauer et al., 1999; Bock et al., 2001; Muellbacher et al., 2002; Tong et al., 2002; Miall et al., 2004).

Interference between opposing visuomotor rotations experienced in close temporal proximity during reaching tasks has also been shown (Krakauer et al., 1999; Wigmore et al., 2002). Similar interference has been reported for a throwing task performed with left and right deviating prisms (Goedert and Willingham, 2002). However, unlike the force-field learning, retrograde interference was still observed when the left and right prisms were experienced 1 day apart. Thus, the consolidation hypothesis was not supported.

Given these disparate results, it seems critical to further explore and test the consolidation hypothesis in relation to visuomotor and dynamic learning. Here, we report the results from six experiments performed in three laboratories. In the first two experiments, we examined whether the lack of consolidation seen in throwing under prisms also applies to the often-studied targetdirected reaching tasks under visuomotor rotations (Krakauer et al., 1999, 2000; Tong et al., 2002; Miall et al., 2004). In the third experiment, we tested whether the consolidation reported for velocity-dependent force fields is also seen in another dynamic 
Table 1. Descriptions of the six experiments

\begin{tabular}{|c|c|c|c|c|c|c|c|c|c|c|c|c|c|}
\hline \multirow{2}{*}{$\begin{array}{l}\text { Location } \\
\text { Kingston }\end{array}$} & \multirow{2}{*}{$\begin{array}{l}\text { Perturbation } \\
\text { Visuomotor rotation }\end{array}$} & \multirow{2}{*}{$\begin{array}{l}\text { Movement } \\
\text { Out and back }\end{array}$} & \multirow{2}{*}{$\begin{array}{l}\text { Posture } \\
\text { Supported }\end{array}$} & \multirow{2}{*}{$\begin{array}{l}\text { Experiment } \\
1\end{array}$} & \multirow{2}{*}{$\begin{array}{c}\text { Subjects } \\
7\end{array}$} & \multirow{2}{*}{$\begin{array}{l}\text { Group } \\
\text { Control }\end{array}$} & \multicolumn{2}{|c|}{ Paradigm } & \multirow[b]{2}{*}{ A } & \multirow[b]{2}{*}{ (1) } & & \multirow[b]{2}{*}{ (1) } & \multirow[b]{2}{*}{$A$} \\
\hline & & & & & & & & & & & & & \\
\hline & & & & & 8 & Test & & & $A$ & (1) & B & (1) & $A$ \\
\hline & & & & 2 & 5 & Control & & & NA & (1) & NA & (1) & NA \\
\hline & & & & & 8 & Test & & & NA & (1) & NB & (1) & NA \\
\hline \multirow[t]{5}{*}{ London } & Position-dependent force field & Out and back & Free & 3 & 6 & Control & & & $A$ & (1) & & (1) & $A$ \\
\hline & & & & & & & & & A & & & & \\
\hline & & & & & 6 & Test 5 min & & & B & (1) & & (1) & A \\
\hline & & & & & 6 & Test $24 \mathrm{hr}$ & & & A & (1) & B & (1) & A \\
\hline & & & & & & & & & A & & & & \\
\hline \multirow[t]{7}{*}{ Kingston } & Velocity-dependent force field & Out and back & Free & 4 & 6 & Test 5 min & & & B & (1) & A & (1) & \\
\hline & & & & & 11 & Control $24 \mathrm{hr}$ & & & A & (1) & & (1) & A \\
\hline & & & & & 8 & Test $24 \mathrm{hr}$ & & & A & (1) & B & (1) & A \\
\hline & & & & & 5 & Control 1 week & & & A & (7) & & (7) & A \\
\hline & & & & & 8 & Test 1 week & & & A & (7) & B & (7) & A \\
\hline & & Point to point & Supported & 5 & 9 & Control & $\mathrm{N}$ & (1) & A & (1) & & (1) & A \\
\hline & & & & & 9 & Test & $\mathrm{N}$ & (1) & A & (1) & $B$ & (1) & A \\
\hline \multirow[t]{2}{*}{ Kyoto } & Velocity-dependent force field & Point to point & Supported & 6 & 6 & Control & $\mathrm{N}$ & (1) & NA & (1) & NN & (1) & NA \\
\hline & & & & & 9 & Test & $\mathrm{N}$ & (1) & NA & (1) & NB & (1) & NA \\
\hline
\end{tabular}

(1) , 1d; (7), $7 \mathrm{~d}$; N, null trials; A, perturbation trials; B, opposite perturbation to $A$.

task; namely, a position-dependent force field. In the remaining three experiments, we tested interference under velocitydependent force fields in a number of conditions.

We failed to observe consolidation in any of these experiments. That is, we found complete interference between opposing visuomotor rotations, opposing position-dependent force fields, and opposing velocity-dependent force fields under a variety of tasks, even when the fields were experienced $\geq 24 \mathrm{hr}$ apart.

\section{Materials and Methods Subjects}

Experiments were run in Kingston, Canada; Kyoto, Japan; and London, United Kingdom. In total, 120 right-handed subjects participated after providing informed consent, and all experimental protocols were approved by local ethics boards. All subjects were naive to the experimental hypotheses and received payment for their participation. All subjects had normal or corrected for normal vision and had no obvious motor deficits. Three subjects were later removed from the analysis because they failed to show normal adaptation.

\section{Apparatus}

Similar experimental setups were used in Kingston, Kyoto, and London. All required subjects to make reaching movements with their right hand. The position of the hand was tracked, and visual feedback of hand position (in the form of a cursor) and targets could be displayed in real time. In addition, state-dependent forces could be applied to the hand by a robotic manipulandum, and visual feedback of the hand cursor could be rotated. The position of the hand was stored for off-line analysis. The Kyoto and London setups were described in detail by Gomi and Kawato (1996) and Goodbody and Wolpert (1998), respectively. Here, we describe the basic elements of the setups, highlighting key differences.

Kingston: experiments 1 and 2. The arm was supported in the horizontal plane by a lightweight brace mounted on air pucks that floated on a cushion of air allowing near-frictionless motion across a tabletop. In experiment 1 , an electromagnetic recording system (Ascension Technologies, Burlington, VT) was used to record the position of the hand at 100 Hz. In experiment 2, hand position was recorded at $1000 \mathrm{~Hz}$ by a lightweight manipulandum (Phantom Haptic Interface 3.0L; Sensable Technologies, Woburn, MA) that subjects held in their hand. Visual feedback of hand position was provided in the plane of movement using a mirrorprojection system that prevented direct viewing of the hand.

London: experiment 3. Subjects held the handle of a lightweight manipulandum (Phantom Haptic Interface 3.0; Sensable Technologies). The manipulandum measured the position of the hand at $1000 \mathrm{~Hz}$ and could generate forces that were servo-controlled at $1000 \mathrm{~Hz}$. Visual feed- back of hand position was provided in three dimensions using a stereoscopic mirror-projection system that prevented direct viewing of the hand (for full details, see Goodbody and Wolpert, 1998). Subjects adopted a natural posture with the elbow below the hand and shoulder.

Kingston: experiments 4 and 5. The same type of manipulandum used in experiment 3 was used to measure the position of the hand $(1000 \mathrm{~Hz})$ and generate forces at the hand (servo-controlled at $1000 \mathrm{~Hz}$ ). Visual feedback of hand position was provided in the plane of movement using a mirror-projection system that prevented direct viewing of the hand. In experiment 4 , subjects adopted a natural posture with the elbow below the hand and shoulder. In experiment 5 , the arm was supported in the horizontal plane by the air puck system described above.

Kyoto: experiments 6. Subjects held the handle of a robotic manipulandum that could exert forces (Gomi and Kawato, 1996). The forearm was supported in the horizontal plane by a freely rotating beam attached to the handle. The position of the hand was sampled at $500 \mathrm{~Hz}$. Visual feedback of hand position (in the form of a cursor) and targets were projected onto a horizontal opaque screen mounted just above the arm.

\section{Procedure}

The general procedure was similar in all experiments (Table 1). With the exception of experiment 4 , subjects made reaching movements from a central start position to one of eight radial targets, and visual feedback of the hand (i.e., cursor) and target was available throughout the movement.

Experiment 1: visuomotor rotations. This experiment included two groups of subjects, a test group $(n=8)$ and a control group $(n=7)$. Participants in the test group performed an out-and-back reaching task with a $+30^{\circ}$ visuomotor rotation imposed on day 1 . Twenty-four hours later, they returned to perform the same task with the opposing $-30^{\circ}$ rotation. After a second $24 \mathrm{hr}$ period, participants performed the task again with the original $+30^{\circ}$ rotation. Participants in the control group performed the task under the $+30^{\circ}$ rotation on day 1 and then again $48 \mathrm{hr}$ later on day 3.

The start circle (diameter, $2.2 \mathrm{~cm}$ ) was located $30 \mathrm{~cm}$ below eye level in the subject's midsagittal plane with the shoulder at $45^{\circ}$ and the elbow at $90^{\circ}$. To initiate a trial, participants moved the cursor (diameter, $1.7 \mathrm{~cm}$ ) into the start circle, at which point one of eight radial targets (diameter, $1.7 \mathrm{~cm}$ ), located $15 \mathrm{~cm}$ from the start, appeared. Participants were instructed to make out-and-back movements (to the target and back to the start) within $500 \mathrm{msec}$. They were encouraged to move smoothly and to make corrections for errors between trials rather than within a trial. This $500 \mathrm{msec}$ time was signaled by both a color change of the start circle and a brief tone. Targets were presented in a sequence starting at $0^{\circ}$ (outward) and continuing counterclockwise (CCW) in $45^{\circ}$ increments. A block was 
defined as a set of eight movements to each target in the sequence. On each testing day, subjects performed 30 blocks ( 240 movements). At the start of the first session, all subjects performed four familiarization blocks (32 movements) in the null condition (i.e., with no rotation).

Experiment 2: visuomotor rotations with washout trials. This experiment included a test group $(n=8)$ and a control group $(n=5)$. The task was the same as in experiment 1, except that subjects performed eight blocks (64 movements) without a visuomotor rotation (null condition) at the beginning of every session. These null or "washout" trials served to washout or remove possible anterograde interference effects from previous learning. Subjects in the test group experienced the same rotation on days 1 and 3 (either $+30^{\circ}$ or $-30^{\circ}$ ) and the opposite rotation on day 2 . The control group experienced the same rotation (either $+30^{\circ}$ or $-30^{\circ}$ ) on all $3 \mathrm{~d}$.

Experiment 3: position-dependent force fields. Eighteen subjects were assigned randomly to one of three groups with six subjects in each. In all groups, on day 1 , subjects first performed the out-and-back reaching task with either a CCW or clockwise (CW) position-dependent rotary force field (Tong et al., 2002). The two test groups then experienced the opposite force field either $5 \mathrm{~min}$ or $1 \mathrm{~d}$ later. All groups returned on day 3 and experienced the same force field as in the first session of day 1 . The stiffness of the position-dependent force field (i.e., the coefficient relating hand displacement from the starting circle to the rotary force applied to the hand) was set at $60 \mathrm{~N} / \mathrm{m}$.

The out-and-back reaching task used was similar to that described for experiment 1 , with the following differences. Auditory feedback warned the subject if they had failed to make the movement in the requisite time of $600 \pm 100 \mathrm{msec}$. Each target would appear once during a block of eight trials but were selected in a pseudorandom order. Each session consisted of 24 blocks, hence 192 trials per session. At the end of every six blocks, the subject was given a $1 \mathrm{~min}$ rest period to avoid fatigue. Before the first session, subjects were given four blocks in a null field (no forces applied), to familiarize themselves with the equipment.

Experiment 4: rotary velocity-dependent force fields with natural arm posture. Thirty-eight subjects were assigned randomly to one of five groups. In all groups, subjects first performed the out-and-back reaching task with a CCW velocity-dependent rotary force field (Shadmehr and Brashers-Krug, 1997). The three test groups then experienced the opposite, CW, force field $5 \mathrm{~min}(n=6), 1 \mathrm{~d}(n=8)$, or 1 week $(n=8)$ later, respectively. The $5 \mathrm{~min}$ and $1 \mathrm{~d}$ groups then returned $1 \mathrm{~d}$ later to experience the original CCW field, whereas the 1 week group returned 1 week later to experience the CCW field. In the two control groups, subjects experienced the CCW fields either $2 \mathrm{~d}(n=11)$ or 2 weeks $(n=5)$ apart. The viscosity of the force field (i.e., the coefficient relating hand velocity to rotary force applied to the hand) was set at $8 \mathrm{~N}-\mathrm{s} / \mathrm{m}$.

The same out-and-back reaching task described for experiment 1 was used, and, again, both visual and auditory cues were provided to indicate the desired movement time $(500 \mathrm{msec})$. For each session with a given force field, subjects performed 30 blocks ( 240 movements). At the start of the first session, all subjects performed four familiarization blocks (32 movements) in the null condition (i.e., with no force field).

Experiment 5: rotary velocity-dependent force fields with arm supported. Eighteen subjects participated in this experiment in which the arm was supported in the horizontal plane by air sleds. Both the control group $(n=9)$ and the test group $(n=9)$ experienced 30 blocks of the null field on day 1 , followed by the CCW velocity-dependent force field on days 2 and 4 . The test group also experienced the CW field on day 3 .

In this experiment, subjects made point-to-point reaches as opposed to out-and-back reaches. On every other trial, they moved from the start position to one of four targets $\left(0,45,90\right.$, and $\left.135^{\circ}\right)$ located $10 \mathrm{~cm}$ away. In the following trial, they then moved back to the start position, which served as the target for that trial. Subjects were required to move to the target within $300 \pm 50 \mathrm{msec}$. If participants arrived too early, the target changed color from blue to red, instructing participants to slow down. If participants arrived at the target too late, the target turned green, instructing them to speed up. If participants reached the target within the $100 \mathrm{msec}$ window, the target remained blue and an audible tone was sounded. The viscosity of the force field was set at $6 \mathrm{~N}-\mathrm{s} / \mathrm{m}$.

Experiment 6: rotary velocity-dependent force fields with washout trials.
Fifteen subjects participated and were assigned randomly to control $(n=$ $6)$ and test $(n=9)$ groups. Both the groups were familiarized with the task by performing 32 blocks ( 256 movements) without any force fields [null force field (NF)] on day 1. On day 2, after eight blocks in the NF, a brief period of rest was provided ( $5 \mathrm{~min}$ ), after which subjects were told that the manipulandum would produce rotational force on the hand. Subjects performed 24 blocks (192 movements) during which the CW force field was presented for half of the subjects and a CCW for the other half. The viscosity of the force field was set at $20 \mathrm{~N}-\mathrm{s} / \mathrm{m}$. On day 3 , after eight blocks in the NF and a brief period of rest, the test subjects were told that they would experience a different rotational force on the hand. Test subjects performed 24 blocks in the force field rotating opposite to the one they had experienced on day 2. The control group performed 24 additional blocks of the NF instead of the force field. On day 4 , after eight blocks in the NF and a brief period of rest, subjects were told that they would now experience the same force as on the second day. Subjects again performed 24 blocks in the force field trained on day 2 .

The start circle (diameter, $2 \mathrm{~cm}$ ) was located $45 \mathrm{~cm}$ in front of the shoulder. To initiate a trial, participants moved the cursor (diameter, 0.4 $\mathrm{cm}$ ) into the start circle, at which point one of eight radial targets (diameter, $3 \mathrm{~cm}$ ) appeared located $12.5 \mathrm{~cm}$ from the start. Participants were required to make a movement to the target with a duration (from leaving the start circle) of $500 \pm 50 \mathrm{msec}$. Visual feedback of the hand position was available during movements, and the entire hand path was shown after movement terminated. Each target would appear once during a block of eight trials, but in a pseudorandom order. The force field was off when subjects returned to the start position and was on only during the outward movements.

\section{Analysis}

To quantify learning, we analyzed the trajectories of the hand in the horizontal plane in which targets were presented. In general, a measure was selected to quantify the performance on a single movement, and to assess performance on a particular block, the median of this measure was taken over the set of eight movements that comprise the block.

For visuomotor rotation experiments, we used as a measure the initial angular error of the movement before any visual feedback could be incorporated to adjust the trajectory. This error was computed as the angle between the vector from the start circle to the cursor position $150 \mathrm{msec}$ into the movement and the vector from the start circle to the target. The reaching movement was considered to start when the tangential velocity of the hand last exceeded $10 \mathrm{~cm} / \mathrm{sec}$ before reaching a speed of $40 \mathrm{~cm} / \mathrm{sec}$.

For the position-dependent force fields, we measured the mean perpendicular displacement of the hand from the straight line joining the start circle and target. When subjects first encounter the positiondependent force field, the hand is typically deflected in terms of direction, but the hand path may not be curved. This feature is well captured by a measure of the deviations from a straight-line movement to the target.

For the velocity-dependent force fields, we used an area measure that measures the area enclosed by the trajectory when the first and last points are joined. When subjects first experience a velocity-dependent force field, their hand path is typically curved and, in out-and-back movements, large loops in the path are seen. These features are well captured by a measure of the area enclosed by the hand trajectory.

In addition, we analyzed experiment 6 using the vector correlation measure proposed in Shadmehr and Mussa-Ivaldi (1994). To perform this analysis, we aligned the velocities sampled over $6 \mathrm{msec}$ intervals to the first time the velocity exceeded $2 \mathrm{~cm} / \mathrm{sec}$ and used the next 83 data points $(498 \mathrm{msec})$. We found the average velocity vectors for each direction of movement in the last 32 movements in the day 1 null field. These could be compared with the movements on the other days. There was a typographical error (F. Mussa-Ivaldi, personal communication) in the original formulation of the correlation measure described by Shadmehr and Mussa-Ivaldi (1994), and we therefore provide the corrected formulation and Matlab code in the Appendix.

For all of the above measures, we assessed retention of learning in the $\mathrm{ABA}$ paradigm by comparing the initial performance levels in the first and second sessions of A using repeated-measures ANOVA. 


\section{Expt. 1: Visuomotor Rotation}
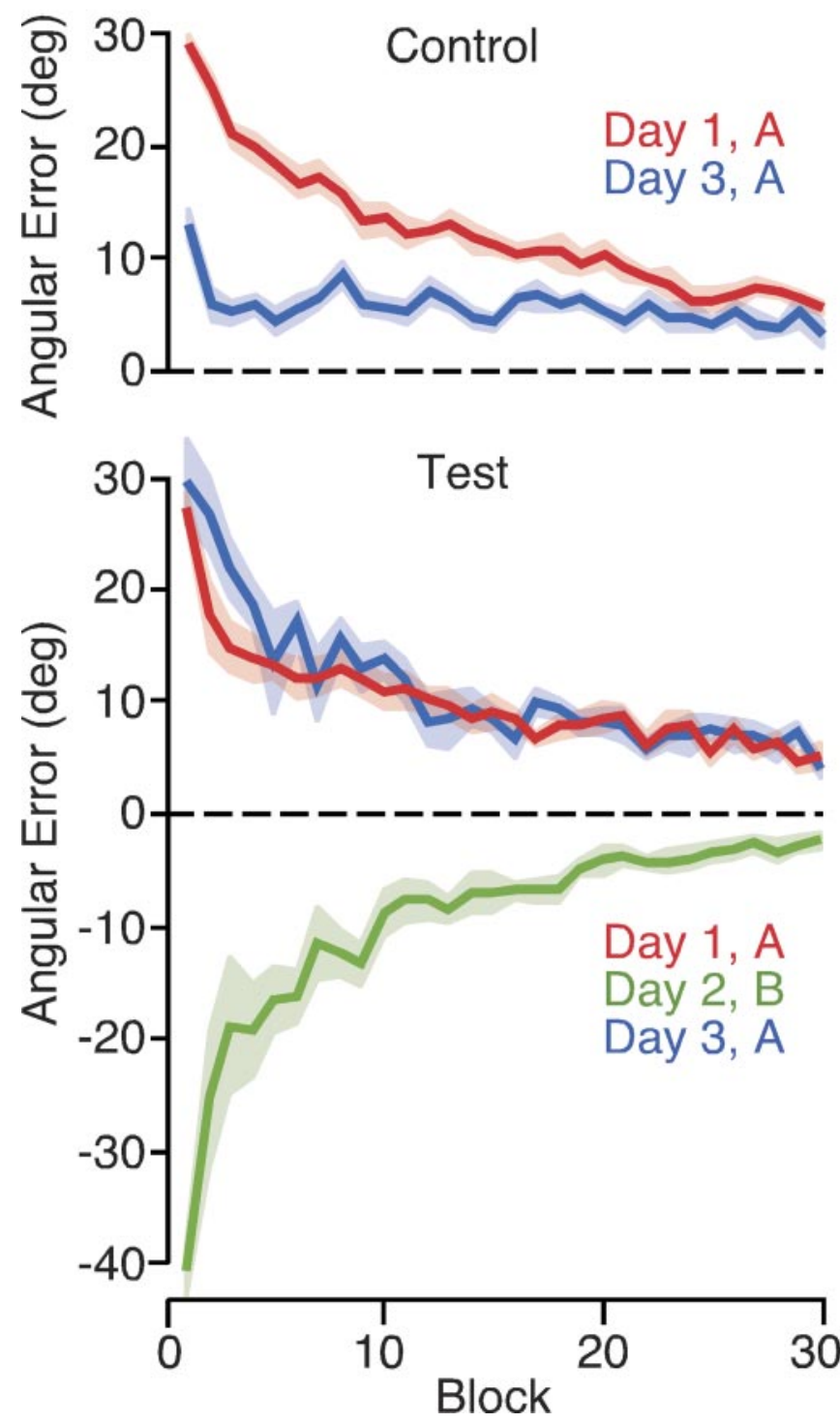

Figure 1. Adaptation to visuomotor rotations. Curves show mean angular error as a function of block on different days. The height of the shaded areas represents $\pm 1 \mathrm{SE}$. For clarity, we have shaded the area formed by joining confidence intervals between adjacent blocks. The top panel shows results for the control group who adapted to the same visuomotor rotation (A) on days 1 and 3. The bottom panels show results for the test group who also adapted to the opposing visuomotor rotation (B) on day 2.

\section{Results}

\section{Experiment 1: visuomotor rotations}

In the first experiment, we tested whether adapting to opposing visuomotor rotations $24 \mathrm{hr}$ apart would interfere with retention of the first rotation. Figure 1 shows learning curves for control and test subjects. The curves show angular error as a function of block, and the ordinate represents the average across subjects in which the value for each subject is the median of the errors of the eight trials within a block. The height of the shaded area represents \pm 1 SE. Subjects in the control group, who experienced the same visuomotor rotation (A) on days 1 and 3, showed clear retention of learning on day 3 . To quantify learning, we computed, for each subject and day, the average angular error across the second and third blocks. This initial angular error was significantly less $\left(F_{(1,6)}=425 ; p<0.001\right)$ on day $3(\mathrm{M}=5.48$; $\mathrm{SE}=$
$0.96)$ than on day $1(M=23.23 ; \mathrm{SE}=0.83)$. Subjects in the test group, who experienced the opposing rotation (B) on day 2, showed no retention and, in fact, were worse on day 3 compared with day 1 . The initial error on day $3(\mathrm{M}=24.5 ; \mathrm{SE}=2.7)$ was significantly greater $\left(F_{(1,7)}=17.3 ; p=0.004\right)$ than on day $1(\mathrm{M}=$ 16.46; $\mathrm{SE}=2.5$ ).

Previous studies have shown that learning of a visuomotor rotation fails to be retained if an opposing visuomotor rotation is experienced 5 min later (Krakauer et al., 1999; Wigmore et al., 2002). In addition, our results are in line with those of Goedert and Willingham (2002), who have recently shown, using a throwing task with prisms, complete interference between opposing visuomotor rotations experienced $24 \mathrm{hr}$ apart. Our results show that this lack of consolidation also applies to the well studied reaching task to radial targets.

\section{Experiment 2: visuomotor rotations with washout trials}

There are two processes at play when we learn two opposing tasks in succession: retrograde interference, the effect of learning the new task on retention of the old task, and anterograde interference, the effect of the old task on the new. The poor performance on day 3 seen in the test group of experiment 1 (Fig. 1), could be attributable to retrograde interference of task $B$ (day 2) on the previously learned task A (day 1) or anterograde interference from task B (day 2) affecting task A on the following day (day 3 ). Indeed, this poor performance could arise from a combination of partial anterograde and retrograde effects (Miall et al., 2004). Experiment 2 was similar to experiment 1, except that subjects performed eight blocks of null trials without a visuomotor rotation at the beginning of each day. These washout trials were included to wash out or remove anterograde effects so as to enable us to test for retrograde effects (Krakauer et al., 2003).

Subjects in the control group experienced the same visuomotor rotation (A) on days 1-3. The washout trials on days 2 and 3 (Fig. 2, null) reveal the presence of initial anterograde effects that gradually washed out. Similar washout effects were seen in the test group. To test whether the washout was complete, we compared, for days 2 and 3 separately, the error at the end of the washout trials (block 8) with the error at the end of the null trials (also block 8) on day 1. The latter provided a baseline because there was no previous adaptation. For the control group, the errors on day $2(\mathrm{M}=-3.15 ; \mathrm{SE}=0.49)$ and day $3(\mathrm{M}=-1.64$; $\mathrm{SE}=0.37)$ were both significantly greater in magnitude $\left(F_{(1,4)}=\right.$ $88.4, p<0.001$ and $F_{(1,4)}=15.9, p=0.016$, respectively) than the error on day $1(\mathrm{M}=-0.74 ; \mathrm{SE}=0.31)$. For the test group, the magnitude of the error on day $2(\mathrm{M}=-2.37$; $\mathrm{SE}=0.52)$ was significantly less $\left(F_{(1,7)}=10.8 ; p=0.013\right)$ than on day $1(\mathrm{M}=$ 0.19 ; $\mathrm{SE}=0.55)$. However, there was no difference $\left(F_{(1,7)}=4.2\right.$; $p=0.08)$ between the error on day $3(\mathrm{M}=1.55$; $\mathrm{SE}=0.55)$ and the error on day 1 . These results indicate that anterograde effects may still have been present after the washout trials. However, it should be emphasized that these effects were very small $\left(<3^{\circ}\right)$.

Subjects in the test group showed no retention and were, in fact, worse on day 3 compared with day 1 : the initial error on day $3(\mathrm{M}=17.9 ; \mathrm{SE}=2.14)$ was significantly greater $\left(F_{(1,7)}=10.9\right.$; $p=0.013)$ than on day $1(\mathrm{M}=14.2 ; \mathrm{SE}=2.12)$. In the control group, initial performance on day $3(\mathrm{M}=3.4 ; \mathrm{SE}=3.26)$ was better than on day $1(\mathrm{M}=12.7 ; \mathrm{SE}=2.24)$, although this effect was only marginally significant $\left(F_{(1,4)}=7.2 ; p=0.055\right)$.

The results of experiment 2 demonstrate that the failure to retain learning of a visuomotor rotation when learning an opposing rotation 1 day later can be explained by retrograde effects. 


\section{Expt. 2: Visuomotor Rotation with Washout}

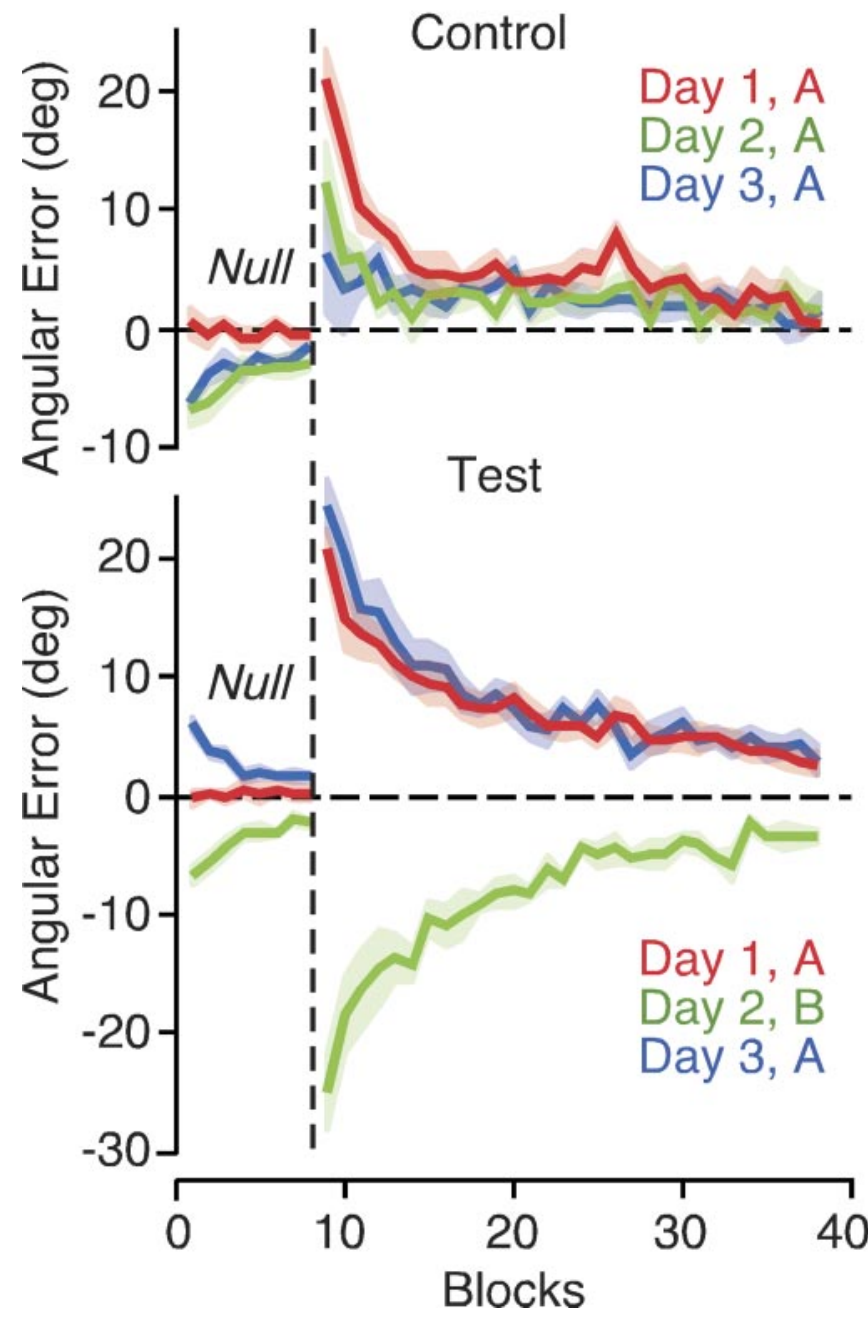

Figure 2. Adaptation to visuomotor rotations with washout trials. Curves show mean angular error as a function of block on different days. Curves to the left of the dashed vertical line show performance in null trials; those to the right show adaptation to the visuomotor rotation. The height of the shaded areas represents \pm 1 SE. The top panel shows results for the control group who adapted to the same visuomotor rotation $(A)$ on days $1-3$. The bottom panels show results for the test group who also experienced $A$ on days 1 and 3 but adapted to the opposing visuomotor rotation (B) on day 2.

\section{Experiment 3: position-dependent force fields}

Experiments 1 and 2, taken together with previous work (Goedert and Willingham, 2002), demonstrate that memory for visuomotor rotations is vulnerable to interference from learning of an opposing rotation even with a $24 \mathrm{hr}$ interval between tasks. This contrasts with previous reports on learning of velocitydependent force fields (Brashers-Krug et al., 1996; Shadmehr and Brashers-Krug, 1997). One possible explanation for this discrepancy is that opposing dynamic tasks (e.g., force fields) can be learned independently, provided they are separated by a time period sufficient for consolidation, whereas opposing kinematic tasks (e.g., visuomotor rotations) cannot. To test whether this putative consolidation applies to other dynamic conditions, in experiment 3, we tested opposing position-dependent rotary force fields (Fig. 3). To quantify performance in this task, we used the mean perpendicular distance from the hand path to the straight line joining the start circle and target.

\section{Expt. 3: Position-Dependent Force Field}

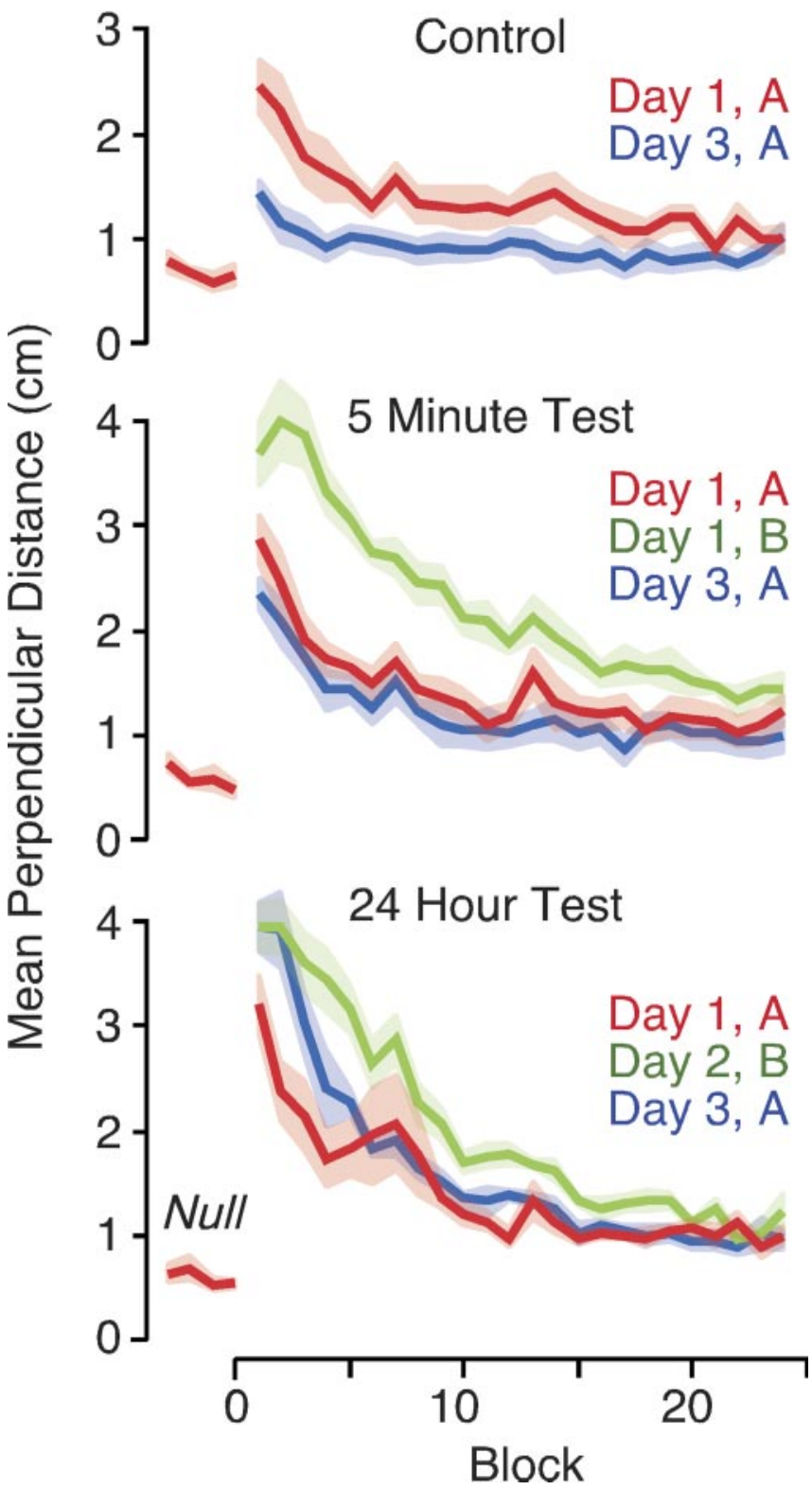

Figure 3. Adaptation to position-dependent force fields. Curves show mean perpendicular distance as a function of block on different days. The height of the shaded areas represents \pm 1 SE. The top panel shows results for the control group who adapted to the same force field $(A)$ on days 1 and 3 . The middle and bottom panels show results for the $5 \mathrm{~min}$ test group and the $24 \mathrm{hr}$ test group who also adapted to the opposing force field (B) either 5 min after $A$ on day 1 or on day 2 , respectively.

Subjects in the control group, who experienced the same position-dependent force field (A) on days 1 and 3, showed clear retention of learning on day 3 . The initial mean perpendicular distance (averaged over the second and third blocks) on day 3 $(\mathrm{M}=1.09 ; \mathrm{SE}=0.153)$ was significantly less $\left(F_{(1,5)}=15.6 ; p=\right.$ $0.011)$ than on day $1(\mathrm{M}=2.01 ; \mathrm{SE}=0.276)$. Subjects in the 5 min test group, who experienced the opposing force field (B) 5 min after encountering the initial force field (A) on day 1 , showed no retention on day 3 compared with day 1 . The initial mean perpendicular distance on day $3(\mathrm{M}=1.92 ; \mathrm{SE}=0.193)$ was not 
Expt. 4: Velocity-Dependent Force Field

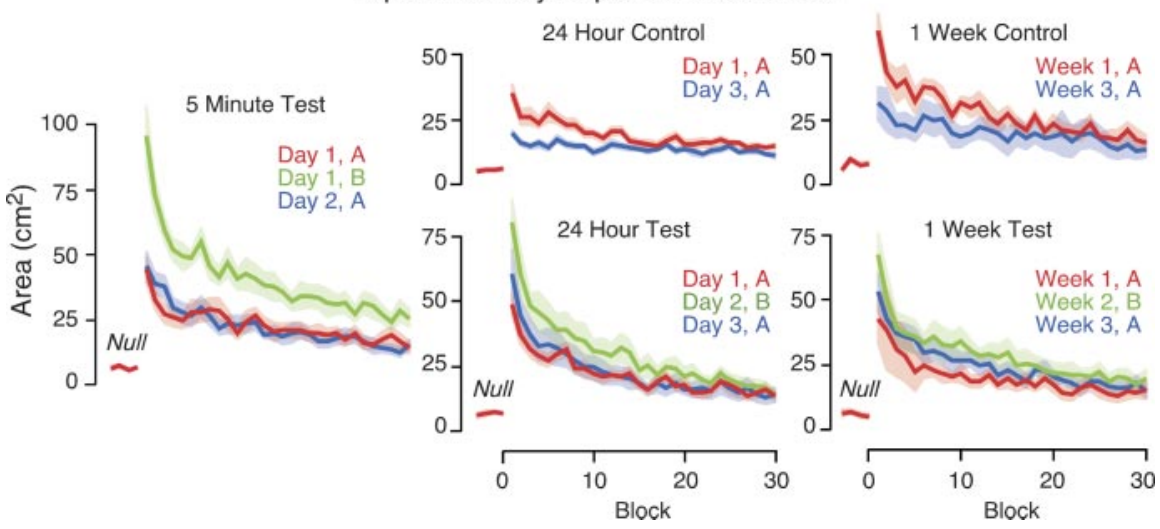

Figure 4. Adaptation to velocity-dependent force fields. Curves show mean area enclosed by the hand path as a function of block on different days. The height of the shaded areas represents $\pm 1 \mathrm{SE}$. The left panel shows results for the 5 min test group who adapted to opposing force fields ( $A$ and then $B$ ) separated by 5 min on day 1 and then were retested on $A$ on day 2 . The middle panels show results for the $24 \mathrm{hr}$ control and test groups who adapted to A on day 1 and were retested on $A 2 \mathrm{~d}$ later. The $24 \mathrm{hr}$ test group also adapted to the opposing force field B on day 2 . The right panels show results for the 1 week control and test groups who adapted to $A$ on day 1 and were retested on $A 2$ weeks later. The 1 week test group also adapted to the opposing force field B a week after first adapting to $A$ on day 1.

reliably different $\left(F_{(1,5)}=0.62 ; p=0.47\right)$ from that on day 1 $(\mathrm{M}=2.19 ; \mathrm{SE}=0.267)$.

Subjects in the $24 \mathrm{hr}$ test group, who experienced the opposing force field (B) $24 \mathrm{hr}$ after encountering the initial force field (A), showed no retention on day 3 compared with day 1 and were, in fact, significantly worse on day 3 . The initial mean perpendicular distance on day $3(\mathrm{M}=3.48 ; \mathrm{SE}=0.32)$ was significantly greater than $\left(F_{(1,5)}=18.1 ; p=0.008\right)$ than on day $1(\mathrm{M}=2.26$; $\mathrm{SE}=$ $0.3)$.

The results of experiment 3 show that people fail to retain learning of a position-dependent force field when subsequently learning an opposing force, even when this subsequent learning occurs 1 day later. More broadly, we can conclude that the previously reported consolidation for opposing velocity-dependent force fields does not indicate that such consolidation is a general property of dynamic tasks.

\section{Experiment 4: velocity-dependent force fields with a natural arm posture}

One key difference between the methodology used in experiment 3 and that used in previous studies showing independent learning of opposing velocity-dependent force fields (Brashers-Krug et al., 1996; Shadmehr and Brashers-Krug, 1997) is the posture of the arm. Whereas the arm was constrained to move in the horizontal plane in studies of velocity-dependent force-field learning, subjects in experiment 3 were free to adopt a natural arm posture. These differences in arm posture certainly affect neural coding of movement (Scott and Kalaska, 1995) and could have an effect on the consolidation process. In experiment 4, we tested for independent learning of opposing velocity-dependent force fields, separated by either $5 \mathrm{~min}, 24 \mathrm{hr}$, or 1 week, when subjects adopted a natural posture for reaching (Fig. 4). To assess performance in this task, in which subjects made movements out from the start circle to the target and back, we used the area enclosed by the hand path.

Subjects in the 5 min test group, who experienced the opposing force field (B) 5 min after encountering the initial force field (A) on day 1 , showed no retention on day 2 compared with day 1 .
The initial area (averaged over the second and third blocks) on day $2(\mathrm{M}=38.5$; $\mathrm{SE}=3.6)$ was reliably greater $\left(F_{(1,7)}=\right.$ $12.2 ; p=0.01)$ than on day $1(\mathrm{M}=30.1$; $\mathrm{SE}=3.6)$. Subjects in the $24 \mathrm{hr}$ control group, who experienced the same velocitydependent force field (A) on days 1 and 3, showed clear retention of learning on day 3. The initial area on day $3(\mathrm{M}=15.4$; $\mathrm{SE}=1.1)$ was significantly less $\left(F_{(1,10)}=\right.$ $14.1 ; p=0.004)$ than on day $1(\mathrm{M}=26.0$; $\mathrm{SE}=3.0)$. Subjects in the 1 week control group, who experienced the same velocitydependent force field (A) 2 weeks apart, showed clear retention of learning in week 3 . The initial area in week $3(\mathrm{M}=25.7$; $\mathrm{SE}=6.4)$ was significantly less $\left(F_{(1,4)}=\right.$ $12.5 ; p=0.02)$ than in week $1(\mathrm{M}=25.7$; $\mathrm{SE}=6.4)$.

Subjects in the $24 \mathrm{hr}$ test group, who experienced the opposing force field (B) $24 \mathrm{hr}$ after encountering the initial force field (A), showed no retention on day 3 compared with day 1 . The initial area on day $3(\mathrm{M}=40.9$; $\mathrm{SE}=6.4)$ was not significantly different $\left(F_{(1,7)}=1.7 ; p=0.23\right)$ from that on day $1(\mathrm{M}=$ 34.4; $\mathrm{SE}=2.9$ ). Subjects in the 1 week test group, who experienced the opposing force field (B) 1 week after the initial force field (A), showed no retention in week 3 compared with week 1 . The initial area in week $3(\mathrm{M}=40.0 ; \mathrm{SE}=4.2)$ was not significantly different $\left(F_{(1,5)}=0.23 ; p=0.66\right)$ from that in week $1(\mathrm{M}=34.9$; $\mathrm{SE}=13.2)$.

The results of experiment 4 show that when people use a natural arm posture, they fail to independently learn opposing velocity-dependent force fields when the tasks are separated by 1 day or even 1 week.

\section{Experiment 5: velocity-dependent force fields with arm supported}

Given our inability to demonstrate independent learning of visuomotor rotations and both position-dependent and velocitydependent force fields, we attempted to replicate more closely the conditions under which such learning has been demonstrated previously (Brashers-Krug et al., 1996; Shadmehr and BrashersKrug, 1997). To this end, in experiment 5, we supported the arm in the horizontal plane, examined point-to-point movements (rather than out-and-back movements as in our previous experiments), tightly constrained movement time to $500 \mathrm{msec}$, and included training in the null field the day before the perturbations were applied (Fig. 5).

Subjects in the control group, who experienced the same force field (A) on days 2 and 4, showed clear retention of learning on day 4 . The initial area on day $4(\mathrm{M}=5.1 ; \mathrm{SE}=0.48)$ was significantly less $\left(F_{(1,8)}=49.2 ; p<0.001\right)$ than on day $2(\mathrm{M}=10.2$; $\mathrm{SE}=0.73)$. Subjects in the test group, who experienced the opposing force field (B) $24 \mathrm{hr}$ after the initial force field (A), showed no retention on day 4 compared with day 2 and, in fact, were worse because the initial area on day $4(\mathrm{M}=13.0$; $\mathrm{SE}=1.4)$ was significantly greater $\left(F_{(1,8)}=12.9 ; p=0.007\right)$ than on day $2(\mathrm{M}=$ 9.1; $\mathrm{SE}=0.6$ ).

The results of experiment 5 failed to replicate the previous studies showing independent learning of opposing velocitydependent force fields when the tasks are separated by 1 day. 


\section{Expt. 5: Velocity-Dependent Force Field with Arm Supported}
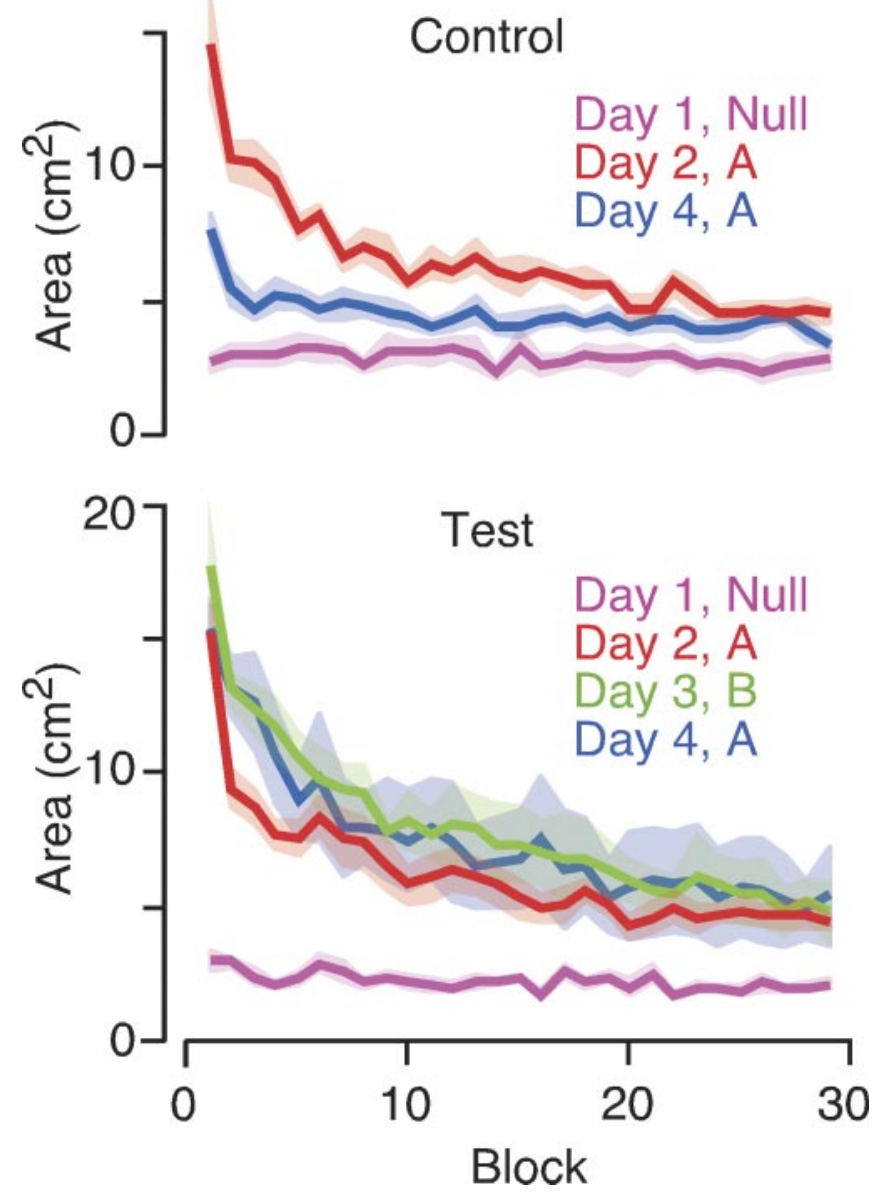

Figure 5. Adaptation to velocity-dependent force fields with the arm supported. Curves show mean area enclosed by the hand path as a function of block on different days. The height of the shaded areas represents $\pm 1 \mathrm{SE}$. The top panel shows results for the control group who trained on null trials on day 1 and then adapted to the same force field $A$ on days 2 and 4 . The bottom panel shows results for the test group who also adapted to the opposing force field B on day 3 .

\section{Experiment 6: velocity-dependent force fields with washout trials}

We performed experiments 3-5 before two recent publications that highlighted the potential importance of including washout trials in removing anterograde effects (Krakauer et al., 2003; Miall et al., 2004). It is unclear whether or not washout trials were included in the previous experiments by Brashers-Krug et al. (1996) and Shadmehr and Brashers-Krug (1997). In any event, we thought it important to test whether we could find retention of learning with washout trials. In experiment 6 , subjects performed a set of null trials a day before perturbations were first applied, and on each day perturbations were tested, they also performed eight blocks of null trials that served as washout trials (Fig. 6). Again, the arm was supported in the horizontal plane, the movements were point to point, and movement time was tightly constrained to be $500 \mathrm{msec}$. To assess task performance, we used the area enclosed by the hand path as well as the velocity vector correlation measure (Shadmehr and Mussa-Ivaldi, 1994) previously used to assess consolidation (Brashers-Krug et al., 1996; Shadmehr and Brashers-Krug, 1997).

Subjects in the control group experienced the same force field
(A) on days 2 and 4 and a full session of null trials on day 3. The washout trials on day 3 (Fig. 6, null) revealed the presence of initial anterograde effects that gradually washed out. Similar washout effects were seen on days 3 and 4 for the test group, who experienced the same force field (A) on days 2 and 4 and the opposing force field (B) on day 3. To test whether the washout was complete, we compared, for days 3 and 4 separately, the error at the end of the washout trials (block 8) with the error at the end of the null trials (also block 8) on day 2. The latter provided a baseline because subjects in both groups experienced only null trials on day 1. For the control group, the area on day $3(\mathrm{M}=3.21$; $\mathrm{SE}=0.47)$ and the area on day $4(\mathrm{M}=2.99 ; \mathrm{SE}=0.20)$ were both not reliably different $\left(F_{(1,5)}=0.01, p=0.913\right.$ and $F_{(1,5)}=0.16$, $p=0.704$, respectively) from the error on day $2(\mathrm{M}=3.14 ; \mathrm{SE}=$ 0.39). Similar results were obtained for the correlation measure. The correlation on day $3(\mathrm{M}=0.948 ; \mathrm{SE}=0.021)$ and the correlation on day $4(\mathrm{M}=0.942 ; \mathrm{SE}=0.022)$ were both not significantly different $\left(F_{(1,5)}=0.17, p=0.702\right.$ and $F_{(1,5)}=0.82, p=$ 0.406 , respectively) from the correlation on day $2(\mathrm{M}=0.939$; $\mathrm{SE}=0.021)$. For the test group, the area on day $3(\mathrm{M}=3.39 ; \mathrm{SE}=$ $0.31)$ and the area on day $4(\mathrm{M}=3.09 ; \mathrm{SE}=0.39)$ were both not significantly different $\left(F_{(1,8)}=0.002, p=0.967\right.$ and $F_{(1,8)}=0.64$, $p=0.448$, respectively) from the error on day $2(\mathrm{M}=3.37$; $\mathrm{SE}=$ $0.23)$. The correlation on day $3(\mathrm{M}=0.953 ; \mathrm{SE}=0.013)$ and the correlation on day $4(\mathrm{M}=0.960 ; \mathrm{SE}=0.008)$ were both not significantly different $\left(F_{(1,8)}=0.15, p=0.712\right.$ and $F_{(1,8)}=1.51$, $p=0.254$, respectively) than the correlation on day $2(\mathrm{M}=0.948$; $\mathrm{SE}=0.009)$. These results indicate that the washout trials were effective in removing anterograde effects.

Subjects in the control group, who experienced the same force field (A) on days 2 and 4 and a full session of null trials on day 3 , did not show retention of learning on day 4 . The initial area on day $4(\mathrm{M}=6.0 ; \mathrm{SE}=0.63)$ was not significantly different $\left(F_{(1,5)}=\right.$ $2.1 ; p=0.21)$ from that on day $2(\mathrm{M}=7.3 ; \mathrm{SE}=0.93)$. This lack of retention was also shown by the correlation measure. The initial correlation (again averaged over the second and third blocks $)$ on day $4(\mathrm{M}=0.854 ; \mathrm{SE}=0.024)$ was not significantly different $\left(F_{(1,5)}=3.3 ; p=0.13\right)$ from that on day $2(\mathrm{M}=0.78$; $\mathrm{SE}=0.044)$. Subjects in the test group, who experienced the opposing force field (B) instead of the null trials on day 3 , also showed no retention on day 4 compared with day 2 . The initial area on day $4(\mathrm{M}=8.1 ; \mathrm{SE}=0.78)$ was no different $\left(F_{(1,8)}=0.53\right.$; $p=0.49)$ than on day $2(\mathrm{M}=7.7 ; \mathrm{SE}=0.62)$. Again, this lack of retention was also shown by the correlation measure. The initial correlation on day $4(\mathrm{M}=0.86 ; \mathrm{SE}=0.02)$ was not significantly different $\left(F_{(1,8)}=0.06 ; p=0.82\right)$ than on day $2(\mathrm{M}=0.851 ; \mathrm{SE}=$ $0.014)$.

The results of experiment 6 not only failed to replicate previous studies showing independent learning of opposing velocitydependent force fields separated by 1 day but also showed that an extended session of null trials could abolish previous learning. That is, not only did learning the opposite force field interfere with learning, practice under normal conditions without forces in the same task also interfered.

\section{Discussion}

We have shown that when people adapt to successive and opposing sensorimotor transformations, they fail to consolidate learning of the first even when the two transformations or tasks are separated by $\geq 24 \mathrm{hr}$. That is, when retested on the first task, performance was no better than when they first encountered the task, and thus they failed to exhibit retention of learning. This result held for both visuomotor rotations and force fields (both 


\section{Expt. 6: Velocity-Dependent Force Field with Washout}

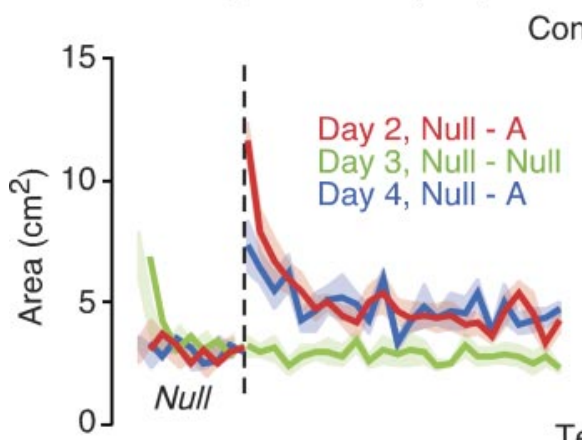

Control
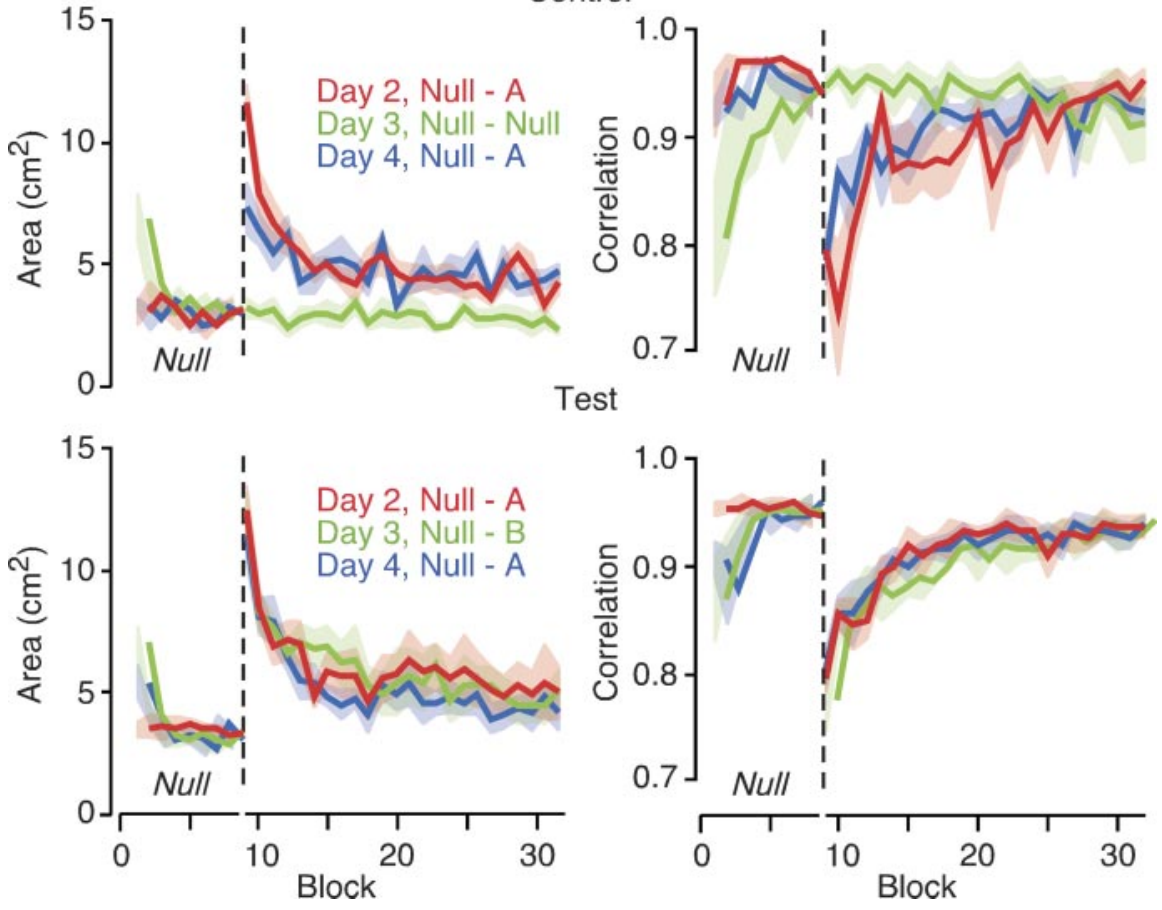

Test

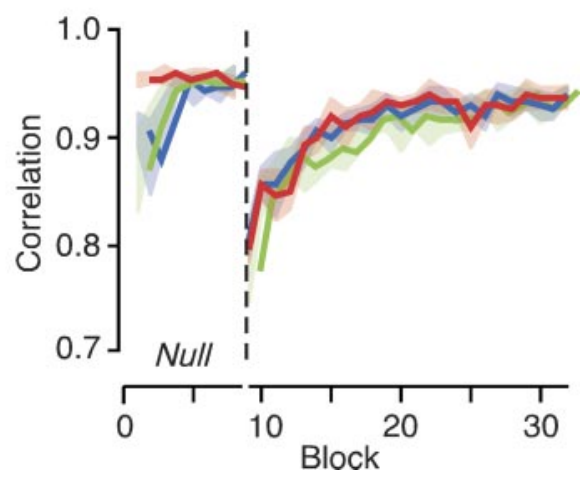

Figure 6. Adaptation to velocity-dependent force fields with the arm supported. Curves in the left panels show mean area enclosed by the hand path as a function of block on different days. Curves in the right panels show the vector correlation measure (see Materials and Methods for details) as a function of block on different days. Curves to the left of the dashed vertical line show performance in null trials performed at the start of each session. The height of the shaded areas represents $\pm 1 \mathrm{SE}$. The top panel shows results for the control group who adapted to the same force field $A$ on days 2 and 4 and experienced a full set of null trials on day 3 . The bottom panel shows results for the test group who adapted to $A$ on days 2 and 4 and the opposing force field $B$ on day 3. Both groups trained on a full set of null trials on day 1 (data not shown).

position and velocity dependent) applied to the hand, two commonly used tasks in studies of human motor adaptation. Not only did subjects fail to retain learning, in several experiments their performance on retest was worse than on the first test. Moreover, failure to consolidate learning was seen not only when subjects subsequently performed the opposing task but also when they performed an equivalent, and large, number of null trials (experiment 6).

It has been suggested that poor initial performance on the retest of $\mathrm{A}$ in the $\mathrm{ABA}$ paradigm may result from anterograde interference of $\mathrm{B}$ on the second experience of $\mathrm{A}$, rather than retrograde interference (Miall et al., 2004). That is, this poor performance could simply arise because subjects expect the opposing transformation B when retested on A. If we assume that only anterograde interference is at play, then we should see retention of learning once the effects of this interference are washed out by adding null trials at the start of each session. However, retention of A was still not observed when these washout trials were included. This result, which is consistent with a recent report on visuomotor learning (Krakauer et al., 2003) and which we have shown for both visuomotor and force-field learning, argues for the presence of retrograde interference.

Our results clearly disagree with the notion that motor memories, at least of novel sensorimotor transformations, undergo a consolidation period after which they are protected against new learning. Instead, they are consistent with the emerging view that emphasizes active and inactive memory states rather than labile and consolidated states [Lewis, 1979; for recent reviews, see Nader et al. (2000) and Nader (2003)]. According to this view, new memories transfer from an initial active state to an inactive state but then can be returned to the active state when needed. Importantly, when reactivated, these memories can be modified and then restored in their modified form to the inactive state. Our results are consistent with the idea that, when people successively encounter opposing transformations (A then B) of the same type (e.g., visuomotor rotations or force fields), memories related to $A$ are reactivated and then modified while adapting to B. Memories related to A are, likewise, reactivated and modified even when null trials are experienced after A. In other words, when people perform the same reaching task that they have previously performed in the same apparatus, they may recall or reactivate motor memories developed from that previous experience without distinguishing between opposing transformations or even between perturbation and null trials. Moreover, the concept of active and inactive states is consistent with the lack of interference seen in our control conditions in which subjects perform A, followed later by A with no intervening task. That is, merely moving around and interacting with the world between the two sessions may be insufficient to transfer the memory of A from an inactive to active state.

The ability to reactivate and modify memories enables skill improvement over multiple practice sessions. For example, if you were to sign up for a series of tennis lessons to be taken once per week, you would want to be able to recall (at least some of) what you had learned the previous week, improve on this learning, and then restore the improved version for subsequent recall. It would presumably be both inefficient and potentially detrimental to store multiple, protected memories pertaining to previous skill levels. Although this reactivation strategy may be detrimental in the current experiments using opposing transformations, it would likely be advantageous when the task being mastered stays the same or becomes elaborated across sessions.

Recent studies have examined motor memory consolidation in the context of sequence learning (Goedert and Willingham, 2002; Walker et al., 2003). Using a serial reaction time task that measures implicit learning of sequences, Goedert and Willingham (2002) found complete interference between different sequences (A and B) experienced $24 \mathrm{hr}$ apart. That is, the usual reduction in reaction time seen when a given sequence $(A)$ is experienced on day 1 and day 3 was not observed when sequence $\mathrm{B}$ was experienced on the intervening day. Although this result suggests that sequence A was not consolidated within $24 \mathrm{hr}$, these researchers did not include a washout period (training with random sequences) and so did not control for possible anterograde interference effects.

Using a finger-tapping task in which subjects were required to continuously press a sequence of four buttons mapped onto the fingertips of one hand, Walker et al. (2003) showed that if subjects practice two different sequences (A and B) $24 \mathrm{hr}$ apart, retention of skilled performance on A (as judged by movement 
speed) is not interfered with. However, if, on day 2, subjects practice (and hence recall) A before performing $B$, then retention of skilled performance on A is not observed. They suggested that the rehearsal of A on day 2 transforms the memory for A into a labile state that is vulnerable to interference from training on B.

The apparent discrepancy between the results of Walker et al. (2003) and our own (i.e., the dependence of retrograde interference on rehearsal of A immediately before performing B) may be attributable to differences in contextual priming. Whereas in the finger-tapping task, rehearsal is required to reactivate memory related to $A$, in the reaching task we examined, the experimental context (i.e., the apparatus and task) appeared to be sufficient. Evidence for such contextual priming in the reaching task comes from the anterograde interference we observed in washout trials (experiments 2 and 6). The anterograde interference suggests that subjects reactivated memory of the previous force-field or visuomotor rotation when placed back in the same context and task. In any event, both our results and those of Walker et al. (2003) challenge the notion of protected motor memories.

It is unclear to us why our results are discrepant with those observed in previous studies on learning opposing velocitydependent force fields (Brashers-Krug et al., 1996; Shadmehr and Brashers-Krug, 1997). First, it is worth emphasizing that the magnitude of the effects previously reported is quite small. Although these studies report some retention for force field A (which increased with the time period between experiencing force fields $\mathrm{A}$ and $\mathrm{B}$ ), retention was far from complete even when $\mathrm{A}$ and $\mathrm{B}$ were separated by $24 \mathrm{hr}$ (Shadmehr and Brashers-Krug, 1997). That is, the level of retention did not reach the level observed in control subjects who did not adapt to force field B. In an effort to identify the source of the discrepancy, we progressively altered a number of factors, across experiments, to more closely match the conditions used by the previous studies. These factors included the orientation of the arm (horizontal vs natural), the movement task (point to point vs center, out and back), the type of force field (position dependent vs velocity dependent), and control of anterograde interference effects (washout trials vs no washout trials). We also used several different dependent measures of performance, including the same correlation measure used in the previous work. However, in no case were we able to find evidence of consolidation of A when followed by B $24 \mathrm{hr}$ later. It may be that subtle differences between the protocols used in the current and previous studies account for the discrepancy in the results. For example, in our experiments, participants received visual feedback about hand position but could not see their arm, whereas in the previous experiments, subjects could see their arm. Another possibility is that subjects in the previous experiments improved the timing of their movements across sessions and that this led to an improvement in the correlation measure that is sensitive to both spatial and temporal features of the trajectory. Nevertheless, although it may be possible to demonstrate small levels of consolidation for learning opposing tasks, we conclude that the effect is not robust.

Using visuomotor rotations and an AABA paradigm, Krakauer et al. (2003) have recently reported that, with extended training on A before (less extensive) training on $\mathrm{B}$, full retention of A is observed. However, it is unclear at present whether this retention is attributable to true consolidation or insufficient training on B given the over-learning of A.

Finally, we would stress that the modifiability of motor memories, or lack of consolidation, reported here is presumably task specific. In particular, the interference we observed between op- posing visuomotor or dynamic perturbations does not imply that, with sufficient changes in the task or task environment, interference cannot be attenuated or eliminated (Wang and Sainburg, 2003; Osu et al., 2004). A key question for future studies is to understand the conditions that lead to previously stored memories being recalled and potentially modified.

\section{Appendix}

Here, we provide the vector correlation measure proposed by Shadmehr and Mussa-Ivaldi (1994). For two scalars, measures $x$ and $y$, we can compute the standard correlation coefficient as follows:

$$
\operatorname{Corr}(x, y)=\frac{\operatorname{Cov}(x, y)}{\sqrt{\operatorname{Var}(x) \operatorname{Var}(y)}}
$$

where $\operatorname{Cov}(x, y)=E(x y)-E(x) E(y), \operatorname{Var}(x)=E\left(x^{2}\right)-[E(x)]^{2}$, $\operatorname{Var}(y)=E\left(y^{2}\right)-[E(y)]^{2}$, and $E()$ represents the expected value or mean of the measure in the bracket. To compute a correlation coefficient between two vector measures, $\mathbf{x}$ and $\mathbf{y}$, we replace multiplications in the above definition with dot products $(\cdot)$ to give the following:

$$
\operatorname{Corr}(\mathbf{x}, \mathbf{y})=\frac{\operatorname{Cov}(\mathbf{x}, \mathbf{y})}{\sqrt{\operatorname{Var}(\mathbf{x}) \operatorname{Var}(\mathbf{y})}}
$$

where $\operatorname{Cov}(\mathbf{x}, \mathbf{y})=E(\mathbf{x} \cdot \mathbf{y})-E(\mathbf{x}) \cdot E(\mathbf{y}), \operatorname{Var}(\mathbf{x})=E(\mathbf{x} \cdot \mathbf{x})-$ $E(\mathbf{x}) \cdot E(\mathbf{x}), \operatorname{Var}(\mathbf{y})=E(\mathbf{y} \cdot \mathbf{y})-E(\mathbf{y}) \cdot E(\mathbf{y})$.

This method corrects a typographical error in the formulation in the appendix of Shadmehr and Mussa-Ivaldi (1994), in which their definition of the SD of the vector measure was incorrect. Specifically, their definition of the SD of a vector measure, $U$, was $\sigma(U)=E(\|U-E(U)\|)^{1 / 2}$ and should instead be $\sigma(U)=E(\| U-$ $\left.E(U) \|^{2}\right)^{1 / 2}$, where $\|U\|=(U \cdot U)^{1 / 2}$.

The Matlab code that performs the vector correlation is given below in which $u$ and $v$ are two $n$-by- 2 matrices representing two different velocity profiles. The columns of the matrices are the two components of the two-dimensional velocity, and the rows represent $n$ velocities sampled over the duration of the movement.

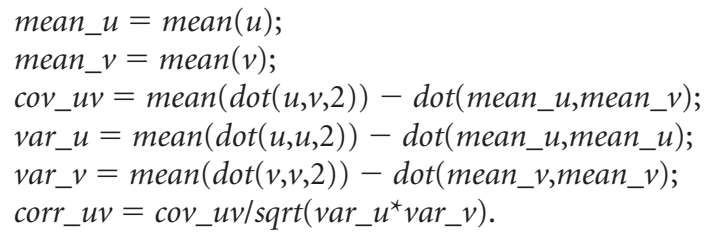

\section{References}

Bock O, Schneider S, Bloomberg J (2001) Conditions for interference versus facilitation during sequential sensorimotor adaptation. Exp Brain Res 138:359-365.

Brashers-Krug T, Shadmehr R, Bizzi E (1996) Consolidation in human motor memory. Nature 382:252-255.

Goedert KM, Willingham DB (2002) Patterns of interference in sequence learning and prism adaptation inconsistent with the consolidation hypothesis. Learn Mem 9:279-292.

Gomi H, Kawato M (1996) Equilibrium-point control hypothesis examined by measured arm stiffness during multijoint movement. Science 272:117-120.

Goodbody SJ, Wolpert DM (1998) Temporal and amplitude generalization in motor learning. J Neurophysiol 79:1825-1838.

Krakauer JW, Ghilardi MF, Ghez C (1999) Independent learning of internal models for kinematic and dynamic control of reaching. Nat Neurosci 2:1026-1031.

Krakauer JW, Pine ZM, Ghilardi MF, Ghez C (2000) Learning of visuomo- 
tor transformations for vectorial planning of reaching trajectories. J Neurosci 20:8916-8924.

Krakauer J, Ghilardi M, Silberstein G, Ghez C (2003) Interference or consolidation? It depends on how much you learn initially. Soc Neurosci Abstr 29:822.819.

Lewis DJ (1979) Psychobiology of active and inactive memory. Psychol Bull 86:1054-1083.

Miall RC, Jenkinson N, Kulkarni K (2004) Adaptation to rotated visual feedback: a re-examination of motor interference. Exp Brain Res 154:201-210.

Muellbacher W, Ziemann U, Wissel J, Dang N, Kofler M, Facchini S, Boroojerdi B, Poewe W, Hallett M (2002) Early consolidation in human primary motor cortex. Nature 415:640-644.

Nader K (2003) Memory traces unbound. Trends Neurosci 26:65-72.

Nader K, Schafe GE, LeDoux JE (2000) The labile nature of consolidation theory. Nat Rev Neurosci 1:216-219.

Osu R, Hirai S, Yoshioka T, Kawato M (2004) Random presentation enables subjects to adapt to two opposing forces on the hand. Nat Neurosci 7:111-112.

Scott SH, Kalaska JF (1995) Changes in motor cortex activity during reach- ing movements with similar hand paths but different arm postures. J Neurophysiol 73:2563-2567.

Shadmehr R, Brashers-Krug T (1997) Functional stages in the formation of human long-term motor memory. J Neurosci 17:409-419.

Shadmehr R, Holcomb HH (1997) Neural correlates of motor memory consolidation. Science 277:821-825.

Shadmehr R, Mussa-Ivaldi F (1994) Adaptive representation of dynamics during learning of a motor task. J Neurosci 14:5:3208-3224.

Tong C, Wolpert DM, Flanagan JR (2002) Kinematics and dynamics are not represented independently in motor working memory: evidence from an interference study. J Neurosci 22:1108-1113.

Walker MP, Brakefield T, Hobson JA, Strickgold R (2003) Dissociable stages of human memory consolidation and reconsolidation. Nature 425:616-620.

Wang J, Sainburg RL (2003) Limitations in interlimb transfer of visuomotor rotations. Exp. Brain Res 149:520-526.

Wigmore V, Tong C, Flanagan JR (2002) Visuomotor rotations of varying size and direction compete for a single internal model in motor working memory. J Exp Psychol Hum Percept Perform 28:447-457. 